\title{
EL COMPLEJO LONICERA PILOSA (KUNTH) SPRENG. (CAPRIFOLIACEAE)
}

\author{
José Á. Villarreal-Quintanilla ${ }^{1,4}$ Alma D. Ruiz-Acevedo ${ }^{1}$, A. Eduardo Estrada- \\ CASTILlÓN $^{3}$ Y Diana JASSO DE RODRÍGUEZ ${ }^{2}$ \\ ${ }^{1}$ Universidad Autónoma Agraria Antonio Narro, Departamento de Botánica, \\ Saltillo, Coahuila, México. \\ ${ }^{2}$ Universidad Autónoma Agraria Antonio Narro, Departamento de \\ Fitomejoramiento, Saltillo, Coahuila, México. \\ ${ }^{3}$ Universidad Autónoma de Nuevo León, Facultad de Ciencias Forestales, \\ Linares, Nuevo León, México. \\ ${ }^{4}$ Autor para la correspondencia: javillarrea100@hotmail.com
}

\section{RESUMEN}

Se revisa el complejo de Lonicera pilosa (Kunth) Spreng., formado por Lonicera cerviculata, L. pilosa var. pilosa y L. pilosa var. glabra. Se realizó un análisis de escalamiento multidimensional utilizando 38 caracteres morfológicos y 152 pliegos de herbario, encontrando que el complejo está formado por tres grupos, dos especies y una nueva variedad que aquí se propone.

Palabras clave: análisis multivariado, Caprifoliaceae, Lonicera.

\section{ABSTRACT}

The Lonicera pilosa (Kunth) Spreng complex, which consists of Lonicera cerviculata, L. pilosa var. pilosa and L. pilosa var. glabra, is reviewed. A multidimensional scaling analysis, using 38 morphological characters and 152 herbarium sheets, was applied. The complex is integrated by three groups, two species and a variety here proposed as new to science.

Key words: Caprifoliaceae, Lonicera, multivariate analysis. 


\section{INTRODUCCIÓN}

El género Lonicera está formado por unas 150 especies agrupadas en una serie de secciones que, de acuerdo con Theis et al. (2008), no presentan monofilia. Se distribuye en regiones templadas y montañosas del Hemisferio Norte y muchos de sus elementos son cultivados como plantas de ornato. Este conjunto diverso y complejo ha sido clasificado en dos subgéneros, cuatro secciones y 24 subsecciones, de las cuales cinco tienen representantes en México (subsec. Purpurascentes, Distegiae, Longiflorae, Phenianthi y Cyhpeolae) (Rehder, 1903). Lonicera pilosa fue descrita en 1818 como Caprifolium pilosum por Kunth. Es la especie del género con más amplia distribución en México (Villarreal-Quintanilla, 2000) y la más vistosa por sus inflorescencias colgantes, por su cáliz con un cuello corto a alargado y por sus flores largamente tubulares de color amarillo, anaranjado o anaranjado-rojizo. De acuerdo con la clasificación infragenérica de Rehder (1903), se ubica en la sección Phenianthi, la cual originalmente incluye cinco especies (L. arizonica, L. ciliosa, L. pilosa, L. sempervirens y L. subaequalis), con flores actinomorfas o ligeramente bilabiadas, con tubo giboso o ventricoso, estambres insertos debajo de la parte media del tubo floral y el par de hojas superiores connadas. Dada su amplia distribución, sus flores llamativas y su fácil recolección, existe en los herbarios un gran número de pliegos de representantes de Lonicera y muchos de ellos están identificados con el nombre de L. pilosa. El estudio detallado de estos ejemplares nos permite deducir que bajo este binomio pueden encontrarse varios taxones fuertemente relacionados que forman un grupo taxonómico caracterizado por presentar flores del largo de las bracteólas y el cáliz de cerca de la mitad del largo del ovario, corola de 4-5 cm de largo y estambres inclusos a ligeamente exertos. Con el propósito de clarificar tal situación y en espera de contribuir al mejor conocimiento de este complejo se presenta el siguiente trabajo.

\section{MATERIALES Y MÉTODOS}

Se examinaron cerca de 300 ejemplares (un poco más de la tercera parte son duplicados) depositados en los herbarios ANSM, CHAPA, CIIDIR, ENCB, MEXU y las bases de datos de ASU, IEB, TEX y XAL (Thiers, continuamente actualizado), en los cuales se encuentran las colecciones más representativas del género en el país. La revisión se realizó con el fin de registrar su distribución, así como analizar y comparar la variación morfológica del grupo, para posteriormente llevar a cabo la 
medición y captura de los caracteres seleccionados para el estudio. Con el propósito de definir las similitudes existentes entre los ejemplares del complejo de Lonicera pilosa, se realizó un análisis multivariado de escalamiento multidimensional con el método de Distancia Euclideana (Johnson y Wichern, 2007) en una Matriz de Coeficientes Gower, con base en caracteres morfométricos. Este procedimiento permite mostrar las relaciones de semejanza (proximidad) de los taxa a través de la representación gráfica de relaciones complejas mediante una configuración de puntos (Linares, 2001).

Previamente la matriz de datos se sometió a una correlación con ETA (nominal por intervalo) en el programa SPSS versión 17 (Meulman y Heiser, 2010). El coeficiente ETA indica la correlación entre variables cuantitativas y categóricas (De la Fuente-Fernández, 2011). Dado que los valores de correlación fueron altos (cercanos a 1), se transformaron los datos a coeficientes Gower, lo cual permite determinar el grado de semejanza entre individuos a los cuales se le han medido características cualitativas, cuantitativas (continuas y discretas) y binarias, con el uso del programa R (Gower, 1967; Chauza-López y Villa-Villada, 2011).

Para el análisis se examinaron 152 muestras de herbario disponibles (no se incluyeron duplicados, muestras incompletas o deterioradas), representativas de la diversidad morfológica del grupo y enumeradas como OTUs (Operational Taxonomic Units). Se seleccionaron 19 caracteres categóricos y 19 cuantitativos continuos, considerados de valor informativo (Cuadro 1). Los datos fueron analizados con el método de correlación utilizando el programa Minitab versión 16 (Greenacre, 2007). Las correlaciones se calcularon por Distancias Euclidianas en el programa SPSS versión 20.

Como parte del tratamiento taxonómico se construyó una clave para identificar los taxones. Se elaboraron descripciones morfológicas completas de las especies y especificaciones diagnósticas de las variedades, además de proporcionar información sobre el hábitat, floración y distribución de los elementos reconocidos.

\section{RESULTADOS}

El agrupamiento de los ejemplares analizados guarda relación con la distribución geográfica. En la Figura 1 se observan tres conjuntos distintos, los dos de la izquierda, que muestran mayor vinculación entre sí, son dos de Lonicera pilosa. Un tercer agregado ubicado en la parte derecha de la figura representa a una entidad diferente, que corresponde a L. cerviculata S. S. White (White, 1947), con distribución en el extremo septentrional de la Sierra Madre Occidental. Lonicera pilosa 
Cuadro 1. Caracteres y estados de carácter empleados en el análisis de similitud.

1. Largo del pecíolo.

2. Forma de la hoja: $0=$ oblonga, $1=$ elíptica.

3. Largo de la hoja.

4. Ancho de la hoja.

5. Proporción entre largo y ancho de la hoja.

6. Ápice de la hoja: $0=$ obtuso, $1=$ redondeado.

7. Base de la hoja: $0=$ obtusa, $1=$ redondeada.

8. Textura de la hoja: $0=$ herbácea, $1=$ coriácea.

9. Indumento del envés de la hoja: $0=$ glabro a escasamente pubescente. $1=$ densamente pubescente.

10. Hojas perfoliadas: $0=$ presentes, $1=$ ausentes.

11. Largo de las hojas perfoliadas.

12. Ancho de las hojas perfoliadas.

13. Largo de la inflorescencia.

14. Ancho de la inflorescencia.

15. Largo del pedúnculo.

16. Largo del cáliz.

17. Indumento del tubo del cáliz: $0=$ glabro, $1=$ piloso y glandular.

18. Indumento de los lóbulos del cáliz: $0=$ glabro, $1=$ piloso y glandular.

19. Largo del tubo del cáliz.

20. Largo de los lóbulos del cáliz.

21. Forma de la corola: $0=$ tubular, $1=$ campanulada.

22. Indumento externo de la corola: $0=$ ausente, $1=$ piloso.

23. Indumento interno de la corola: $0=$ ausente, $1=$ piloso.

24. Largo de la corola.

25. Ancho de la corola en la base.

26. Ancho de la corola cerca el ápice.

27. Forma de los lóbulos de la corola: $0=$ elípticos, $1=$ oblongos.

28. Largo de los lóbulos de la corola.

29. Ancho de los lóbulos de la corola.

30. Giba en el tubo de la corola: $0=$ ausente, $1=$ presente.

31. Ensanchamiento cerca de la base del tubo de la corola: $0=$ ausente, $1=$ presente.

32. Posición de los estambres: $0=$ exertos, $1=$ inclusos.

33. Color de la corola: $0=$ anaranjado-rojizo, $1=$ rojo-púrpura.

34. Largo del fruto.

35. Ancho del fruto.

36. Altitud: $0=1300-1250 \mathrm{~m}, 1=1251-3000 \mathrm{~m}$.

37. Latitud: $0=16^{\circ}-22.5^{\circ} \mathrm{N}, 1=22.6^{\circ}-29^{\circ} \mathrm{N}$.

38. Longitud: $0=92^{\circ}-100^{\circ} \mathrm{W}, 1=101^{\circ}-109^{\circ} \mathrm{W}$. 


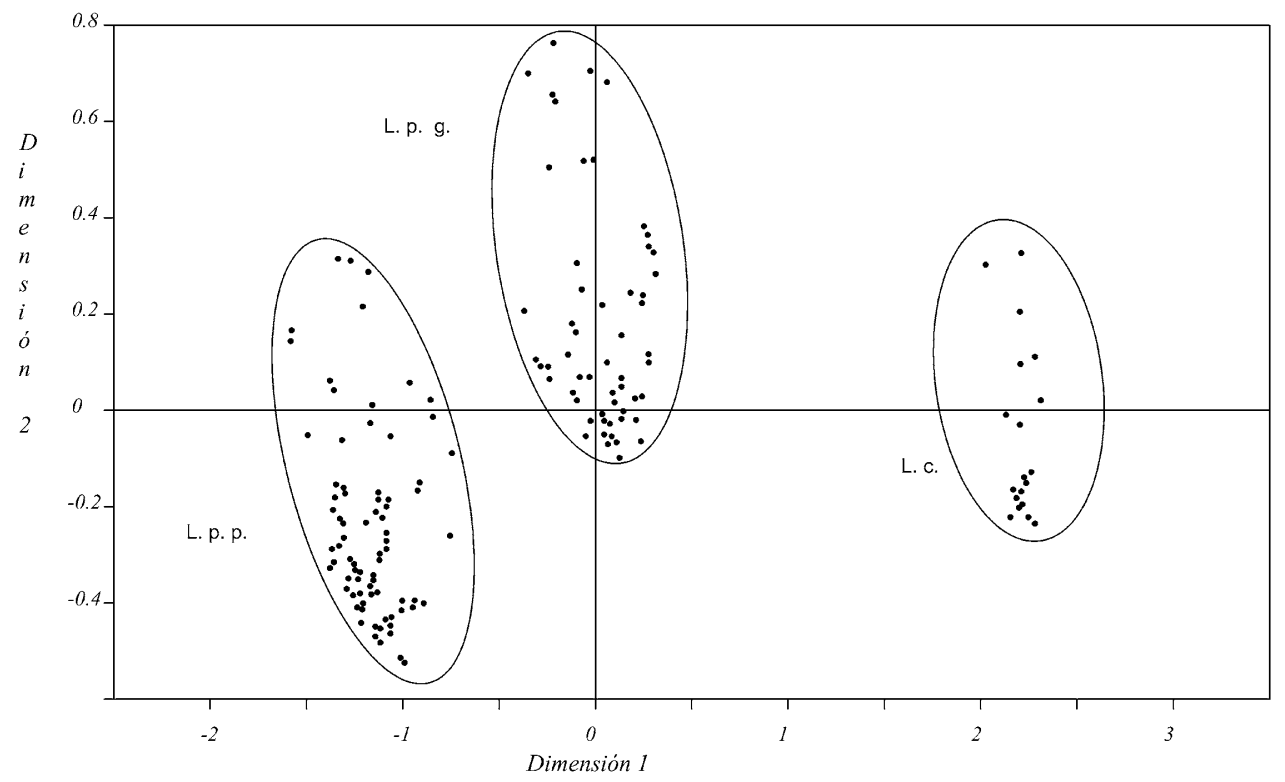

Fig. 1. Gráfica que muestra la agrupación de los representantes del complejo Lonicera pilosa. L.c. (Lonicera cerviculata), L.p.p. (L. pilosa var. pilosa), L.p.g. (L. pilosa var. glabra).

está formada por dos variedades, la típica con una distribución en el oriente, centro y sur del país y otra, aquí propuesta, que habita en el centro y occidente del territorio nacional.

Lonicera cerviculata se conoce, hasta ahora, solo de la región montañosa occidental de Chihuahua y su colindancia con Durango y Sonora. La variedad típica de L. pilosa está registrada en la actualidad en 15 estados del país, en tanto que la nueva se localiza en la Faja Volcánica Transmexicana y en la Sierra Madre Occidental, con representación en 11 entidades políticas del país (Fig. 2). Se definen en este trabajo tres taxones para el complejo, todos ellos endémicos de México.

Clave para la separación de taxones del complejo Lonicera pilosa.

1. Tubo de la corola ensanchado cerca de la base; corola rojiza, con tintes púrpuraanaranjados, de 15-24 mm de largo, glabra internamente; estambres inclusos .... 
1. Tubo de la corola con una giba basal; corola anaranjada, rojiza-anaranjada a amarillenta, de 30-50 mm largo, pilosa internamente; estambres ligeramente exsertos (L. pilosa).

2. Pedúnculo, cáliz y corola con pubescencia pilosa y de pelos glandulares; cuello del cáliz de 0.5-1.0 mm de largo L. pilosa var. pilosa

2. Pedúnculo, cáliz y corola, glabros; cuello del cáliz de 1.5-2.0 mm de largo ........ L. pilosa var. glabra

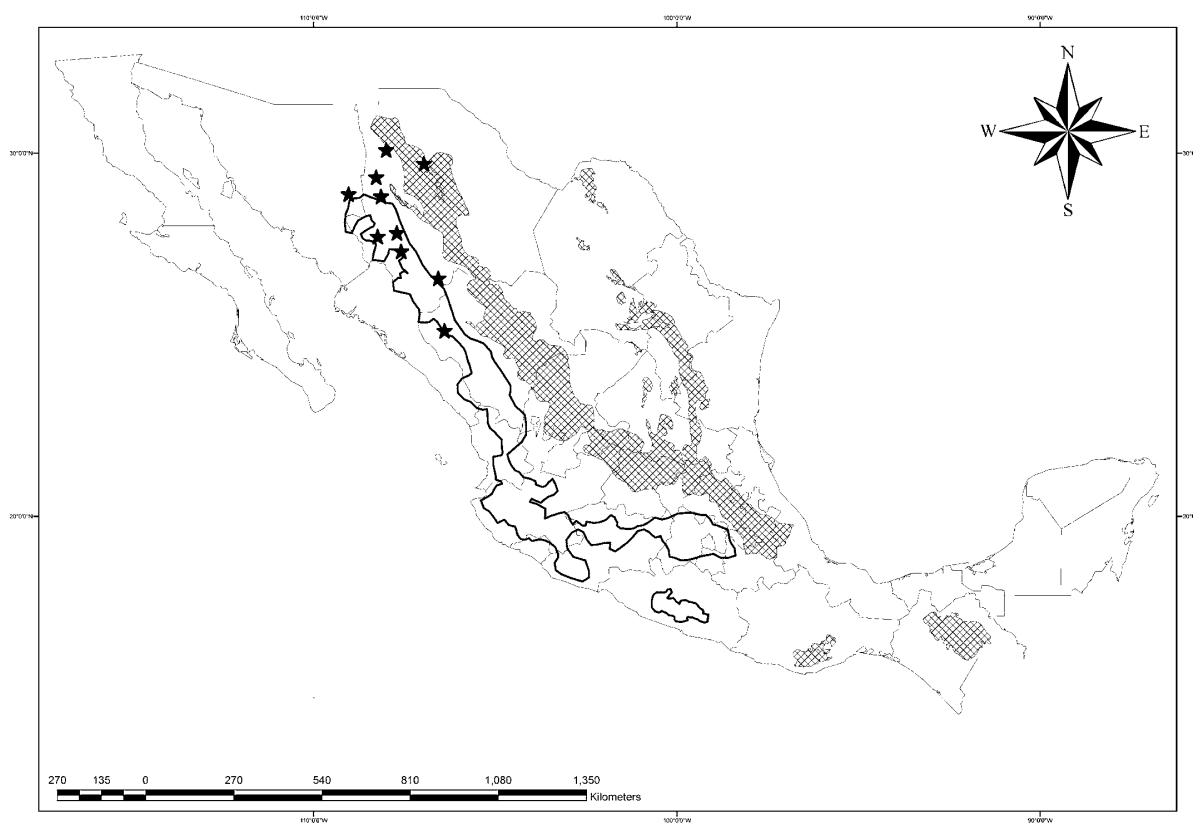

Fig. 2. Distribución conocida de las especies del complejo Lonicera pilosa. Estrellas ( $L$. cerviculata), cuadriculado (L. pilosa var. pilosa) y blanco (L. pilosa var. glabra).

Lonicera cerviculata S. S. White, J. Arnold Arb. 28: 441. 1947. Tipo: MÉXICO. Sonora, Las Tierritas del Temblor, Sierra El Tigre, region of the Rio de Bavispe, 18 ago. 1940, S. S. White 3422 (Holotipo: MICH!).

Arbustos semierectos a volubles, 1-5 m largo; tallos glabros a escasamente pilosos. Hojas con peciolos 2-6 $\mathrm{mm}$ largo, ligeramente pilosos a glabros, láminas 
oblongo-ovadas 3-12 cm largo, 18-45 $\mathrm{mm}$ ancho, ápice obtuso, base redondeada a cuneada, decurrente, margen entero, ligeramente involuto, las de los nudos superiores, anteriores a la inflorescencia, usualmente sésiles, en ocasiones connadoperfoliadas en la base, haz verde y glabro, envés glauco y pubescente, subcoriáceas, discoloras. Flores en espigas terminales, con 1-12 verticilos de 4-8 flores sésiles, glabras; cáliz gamosépalo, pentalobulado, ovoide, 2-3 $\mathrm{mm}$ largo, prolongado en un cuello de 1.0-1.5 mm, lóbulos elípticos a lanceolados, 0.8-1.2 mm largo, soldados en la base; corola gamopétala, pentalobulada, rojiza, con tintes púrpura-anaranjados, tubular a estrechamente campanulada, actinomorfa, 15-24 mm de largo, tubo ensanchado cerca de la base (Fig. 3A), lóbulos elípticos, 3-4 mm de largo; glándula nectarífera en la base ensanchada de la corola; estambres inclusos, filamentos glabros, anteras 4-5 mm largo; pistilo 12-18 $\mathrm{mm}$ de largo, estilo glabro, estigma subcapitado. Fruto globoso, anaranjado-rojizo, $5-8 \mathrm{~mm}$ de diámetro, restos del cáliz persistentes; semillas 3-4, ovoides.

Hábitat. Orilla de arroyos, laderas rocosas, en bosques de pino-encino y de táscates en altitudes de $1400 \mathrm{a} 2200 \mathrm{~m}$.

Floración. Abril-julio.

Distribución. Norte de Sierra Madre Occidental.

Ejemplares examinados. MÉXICO. CHIHUAHUA. Between Maycoba, Sonora and Yepachic, 28 27'27" N, 108 31'10" W, 1524 m, 27 abr. 2002, Baker 14356 (ASU); Mpio. Chihuahua, Parque Cumbres de Majalca, $2120 \mathrm{~m}$, bosque de pinoencino-pastizal, 13 sep. 1997, Estrada \& Yen 7169 (ANSM); Mpio. Guachochi, Norogachi, pine-oak forest, 30 may. 1984, Bye et al. 12817 (MEXU, TEX); Mpio. Madera, Arroyo del Pedernal, Ejido El Lago, Rodríguez 25 (MEXU); Arroyo del Agua, Rodríguez 341 (MEXU); Mpio. Ocampo, Parque nacional Cascada de Basaseachic, $28^{\circ} 10^{\prime}$ N, 108 $12^{\prime}$ W, 1980 m, matorral, 4 oct. 1986, Spellenberg 8842 (MEXU), 11859 (MEXU); Basaseachic falls area, along river, juniper woods, 1950 m, 9 jun. 1976, Pinkava 13266 (ASU); 24 mi up Tinaja rd., Sierra La Brena on Sierra Madre Occidental, Pinus-Quercus wood, 8 jul. 1998, Spencer 680 (TEX); $12 \mathrm{mi}$ E of Pacheco, Sierra La Brena on Sierra Madre Occidental, 3005' N, 108 $21^{\prime}$ W, 2057 m, 10 jun. 1998, Spencer 692 (ASU); 31 mi of Mata Ortíz, south of Pacheco, Sierra La Brena, Sierra Madre Occidental, 30²2' N, 108²2' W, 24 sept. 1988, Spencer 1092 (TEX); Mpio. San Pablo Balleza, 2 km al SW de Agostadero de Aguirre, Cota 7179 
(MEXU, XAL); Mpio. Temosachi, Nabogame, $28^{\circ} 29^{\prime} \mathrm{N}, 108^{\circ} 28^{\prime} \mathrm{W}, 1800 \mathrm{~m}$, oak woodland, 5 may. 1987 King 426 (TEX); Nabogame, 28 30' N, 108³0' W, 1800 m, oak woodland, 21 ago. 1987, Laferrière 426 (ANSM, MEXU), 912 (ANSM, MEXU, TEX), 1665 (CHAPA). DURANGO: Mpio. Canelas, Cuevecillas, cercanía al campamento de la UCODEFO “ Topia”, $2680 \mathrm{~m}$, bosque de pino-encino-abeto, 26 sept. 1990, Bravo 1593 (ANSM, CIIDIR, MEXU); SONORA: N end of Mesa Campanero, Barranca El Salto, 28 $21^{\prime}$ N, 10901' W, 21 jul. 1994, Fishbein et al. 1714 (MEXU, TEX); $1.4 \mathrm{~km} \mathrm{~W}$ of restaurant Puerto de la Cruz on Mex 16, N of Mesa del Campanero, matorral, 26 may. 2004, Van Devender \& Reina 565 (MEXU).

Lonicera pilosa (Willd. ex Kunth) Spreng., Syst. Veg. 1: 178. 1825. Caprifolium pilosum Willd. ex Kunth, Nov. Gen. Sp. (quarto ed.) 3: 427. Tab. 298. 1818. Tipo: MÉXICO. Probablemente Hidalgo, crescit locis subfrigidis Novae Hispaniae, $A$. Humboldt \& A. Bonpland s. $n$. (supuestamente en P, pero sin haberse localizado, por lo que la ilustración puede considerarse como lectotipo).

= Lonicera tubulosa Benth., Pl. Hartw. 37. 1840. Tipo: MÉXICO. Hidalgo, Velasco, near Real del Monte. K.T. Hartweg 292 (Holotipo: E!; isotipo: LD!). L. pilosa (Willd. ex Kunth) Spreng. forma tubulosa (Benth.) Rehder, Ann. Rep. Mo. Bot. Gard. 14: 172. 1903. =L. pilosa (Willd. ex Kunth) Spreng. forma schaffneri Rehder, Ann. Rep. Mo. Bot. Gard. 14: 172. 1903. Tipo: MÉXICO. San Luis Potosí, chiefly in the region of San Luis Potosi, $22^{\circ}$ N, 6000-8000 ft., C.C. Parry \& E. Palmer 297 (Sintipos: MO!, PH); J.W. Schaffner 363 (Sintipos: MO, MICH); A. B. Ghiesbreght 699 (Sintipo: MO).

Arbustos volubles, 4-6 m largo; tallos glabros a pilosos y con pubescencia glandular. Hojas con peciolos 3-12 mm largo, glabros, láminas oblongas, 2-7(-10) cm largo, 20-50 mm ancho, ápice obtuso a redondeado, base redondeada, algo decurrente, margen entero, en ocasiones revoluto, raramente ciliado, el par de hojas superiores, frecuentemente connadas-perfoliadas hasta la mitad de la lámina, haz glabro, envés usualmente pubescente, verde amarillento a glauco, ligeramente discoloras, subcoriáceas. Flores 4-8, en cimas terminales, ocasionalmente en espigas cortas, pedúnculos 2-40 $\mathrm{mm}$ largo, 2-4 $\mathrm{mm}$ ancho, glabros, brácteas, cuando presentes, foliáceas, elípticas, 8-16 mm largo; cáliz gamosépalo pentalobulado, campanulado, 2-3 mm largo, glabro o con pubescencia glandular, lóbulos deltoides, 0.8-1.2 mm largo, soldados, glabros a ciliados, o piloso-glandulares; corola gamopétala pentalobulada, anaranjada, rojiza-anaranjada a amarillenta, tubular, regular, 3-5 cm largo, tubo floral con una giba en la base, lóbulos lanceolados a elípticos, 6-10 mm largo, glabra a pilosa y con pelos glandulares externamente, pilosa internamente, glándula 
nectarífera presente en la giba de la corola; estambres ligeramente exertos, pilosos en la base, anteras $5 \mathrm{~mm}$ largo; pistilo $32-55 \mathrm{~mm}$ de largo, glabro, estigma capitado. Fruto globoso, rosado a rojo, $5-8 \mathrm{~mm}$ de diámetro, con restos del cáliz persistentes: semillas 3-4, ovadas.

Hábitat. Cañadas y lugares húmedos en bosques de pino-encino, de oyamel, mesófilos de montaña, encinares, matorrales y pastizales, en altitudes de 800 a 3200 m.

Floración. Casi todo el año.

\section{Lonicera pilosa (Kunth) Spreng. var. pilosa}

Flores con pedúnculo piloso, cáliz piloso con pelos glandulares, indumento escaso a denso en el tubo, siempre presente en el cuello y lóbulos, cuello 0.5-1.0 $\mathrm{mm}$ largo; corola pilosa y con pelos glandulares externamente (Fig. 3C), y pilosa internamente.

Distribución: Sierras Madres Oriental, Occidental y del Sur (Fig. 2).

Ejemplares examinados: MÉXICO. AGUASCALIENTES: Mpio. Calvillo, 12 km al N del Temazcal, García 4442 (MEXU); Mpio. San José de Gracia, San José de Gracia, 23 jun. 1985, García 2992 (IEB, MEXU); San José de Gracia, 2208' N, $102^{\circ} 24^{\prime}$ W, 8 jul. 1983, Siqueiros 2291 (CIIDIR, IEB). CHIAPAS: Mpio. Comitán de Domínguez, carr. Teopisca-Comitán, frente a Cruz Quemada, Chamé \& Luna 20 (CHAPA, MEXU); Mpio. Chamula, Pathuitz, $16^{\circ} 51^{\prime}$ N, 92 $95^{\circ}$ ' W, 6 jun. 1988, Santiz 888 (CHAPA, TEX); Mpio. Ixtapa, paraje Vo' bit, 1647' N, 9248' W, 17 jun. 1966, Laughlin 1092 (TEX); Mpio. San Cristóbal de las Casas, Peña María, rumbo a San Lucas, 164' N, 92³8' W, 2254 m, 28 jun. 1999, Díaz 54 (ANSM); Col. Carrizal, Mejía \& Luna 432 (MEXU, XAL); Peña María, 1641' N, 92³6' W, 2440 m, 28 jun. 1999, Méndez 47 (ANSM); Mpio. Zinacantán, $600 \mathrm{~m}$ al SW de Chainatic, Mejía \& Luna 499 (MEXU). CHIHUAHUA: Along old railroad westward rancho El Ojito, 5 may 1959, Correll \& Johnston 21495 (ASU, MEXU, TEX); Majalca, 11 may. 1959, Correll \& Johnston 21770 (TEX). COAHUILA: Mpio. Arteaga, predio Santa Anita, Sierra Rancho Nuevo, 252 $26^{\prime}$ N, 100 $33^{\prime}$ W, 2703 m, bosque de pino, 10 sept. 2006, Encina 1457 (ANSM); ladera alta de la Sierra La Veleta, Ejido Nuncio, 251' N, $100^{\circ} 18^{\prime}$ W, 3096 m, bosque de oyamel, 12 jul. 2014, Encina 3801 (ANSM); La Siberia, 2600 m, bosque de pino-encino, 25 jun. 1983, Fernández 1616 (ANSM, CHAPA, 
CIIDIR, MEXU, TEX); Sierra La Viga, 4 mi E of Jame, 2526' N, 100 $35^{\prime} \mathrm{W}$, pine forest, Henrickson et al. 16095 (MEXU, TEX); Sierra Zapalinamé, 2990 m, 19 may. 1990, Hinton 20270 (CHAPA, MEXU, TEX); Los Lirios-El Cercado, pine and oak wood, 2405 m, 29 jul. 1995, Hinton 25425 (ANSM, TEX); Mesa de las Tablas, Núñez

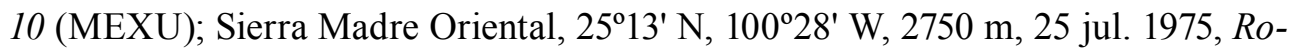
bert \& Passini 15-43-14 (ANSM); Sierra de Arteaga, Cañón La Osamenta, 2520' N, $100^{\circ} 39^{\prime} \mathrm{W}, 2300 \mathrm{~m}$, bosque de pino piñonero, 26 may. 1990, Villarreal \& Carranza 5667 (ANSM, ASU, CHAPA, CIIDIR, MEXU); Mpio. Cuatrociénegas, Sierra de La Madera, $27^{\circ} 02^{\prime} \mathrm{N}, 102^{\circ} 23^{\prime} \mathrm{W}, 20$ sept. 1972, Chiang et al. 9425 (TEX); Sierra La Madera, Cañón La Hacienda, 2703' N, 102²4' W, 2900 m, 5 ago. 1973, Henrickson \& Wendt 11937 (ASU, MEXU, TEX); 6 km de Ostula, Pinus wood, 12 ago. 1976, Henrickson \& Pigge 15285b (MEXU, TEX); road to San Antonio, 2853 m, Douglas fir forest, 21 abr. 1976, Pinkava 13569 (ASU); Sierra La Madera, Cañón El Invierno, 28 ago. 1974, Wendt 645 (TEX); Mpio. Ocampo, Sierra Maderas del Carmen, above Campo Cinco, 17 jun. 1976, Fryxell 2694 (TEX); aserradero Maderas del Carmen, $28^{\circ} 57^{\prime}$ N, 102 $34^{\prime}$ W, 25 may. 1975, Riskind 1780 (TEX); Sierra del Carmen, E of Moreno, Spellenberg 9948 (MEXU); $20 \mathrm{~km} \mathrm{NW}$ of Fraile, Stanford et al. 450 (MEXU); Sierra Maderas del Carmen, Campo Tres, S of canyon El Club and Hell's kitchen, Wendt et al. 484 (MEXU, TEX); Mpio. Ramos Arizpe, Sierra de la Paila, Ejido El Cedral, 1300 m, 25 ago.1992, Valdés 2210 (ANSM); Sierra de La Paila, El Cedral, $26^{\circ} 02^{\prime} \mathrm{N}, 101^{\circ} 23^{\prime} \mathrm{W}, 1300 \mathrm{~m}$, bosque de Quercus, 2 nov. 1988, Villarreal et al. 4952 (ANSM, MEXU), Villarreal et al. 4759 (ANSM); Mpio. Saltillo, Puerto San Lorenzo, 2460 m, bosque de encino, 2 ago. 1979, Arce s. $n$. (ANSM); Cañón de San Lorenzo, 2520' N, 100 $55^{\prime} \mathrm{W}, 2532 \mathrm{~m}$, bosque de encino, 6 jul. 2000, Encina 456 (ANSM, IEB); Sierra de Zapalinamé, Cañón de Santa Rosa, 2519' N, 100 $57^{\prime}$ W, 2427 m, bosque de encino-pino, 11 feb. 2002, Encina 697 (ANSM). DURANGO: Mpio. El Mezquital, Las Cuevitas por el camino a Charcos, $2020 \mathrm{~m}$, bosque de $P i$ nus-Quercus, 11 may. 1987, Acevedo 447 (ANSM, CIIDIR, MEXU); $40 \mathrm{~km}$ de La Escondida, 2740 m, bosque de encino-pino, 18 jun. 1992, González 5152 (ANSM, CIIDIR, MEXU); Mezquital, 18 jul. 1985, González 6263 (CIIDIR, IEB); Mpio. Guanaceví, 40-50 km al E de Guanaceví, Hernández et al. 8331 (MEXU); $12 \mathrm{~km} \mathrm{E}$ of El Salto, 2350' N, 105¹8' W, 23 jun. 1973, Johnston et al. 11444 (TEX); Mpio. Pueblo Nuevo, Corralitos, 26 sept. 1982, Fernández 1187 (CIIDIR, IEB); El Salto, $23^{\circ} 39^{\prime}$ N, 1054' W, 15 jun. 1951, Gentry \& Gilly 10612 (MEXU, TEX); 8-10 km al $\mathrm{S}$ de El Salto, Hernández \& Tenorio 7600 (MEXU); $5 \mathrm{~km}$ de la desviación hacia Chavarria, Tenorio \& Romero 6079 (MEXU); Maíz Gordo, rumbo a Pueblo Nuevo, $23^{\circ} 13^{\prime} \mathrm{N}, 104^{\circ} 56^{\prime} \mathrm{W}, 3034 \mathrm{~m}$, bosque de pino-encino, 23 oct. 2010, Vázquez et al. 59 
(ANSM); Mpio. San Dimas, Mesa del Roble, San Miguel de Cruces, 2700 m, bosque de pino-encino, 20 may. 1990, García \& Acevedo 379 (ANSM, CHAPA, CIIDIR, IEB, MEXU); Mesa del Roble, 26 may. 1990, González \& Acevedo 5008 (CHAPA, CIIDIR, IEB); Mpio. Santiago Papasquiaro, Santiago Papasquiaro, 29 jul. 1990, Benitez 590 (CIIDIR, IEB); km 21 carr. Santiago Papasquiaro -Los Altares, Benitez 1627 (CIIDIR, MEXU); 1.6 mi by Topia, 2504' N, 10549' W, 9 jul. 1983, Corral 226 (TEX); Sierra de las Candelas, $30 \mathrm{mi}$ NW of Santiago Papasquiaro, Straw \& Forman 1770 (MEXU); $14 \mathrm{~km}$ al W de Garame de Abajo, bosque de pino-encino, 15 jul. 1982, Tenorio \& Romero 984 (ANSM, MEXU, XAL); Mpio. Súchil, San Salvador de Ahorcados, 25 oct. 1986, Acevedo 95 (CIIDIR, IEB); Cerro Las Iglesias, Sierra de Urica, $7 \mathrm{~km}$ al E de El Alemán, Reserva de la Biosfera La Michilía, Acevedo 335 (CIIDIR, MEXU); Reserva de la biosfera La Michilía, 23⒉ $21^{\prime} \mathrm{N}, 104^{\circ} 11^{\prime} \mathrm{W}, 2400 \mathrm{~m}$, bosque de Quercus-Pinus, 11 may. 1992, García 1300 (ANSM, CIIDIR); Reserva La Michilía, arroyo San Pedro, García 1660 (CIIDIR, MEXU); San Juan de Michis, $23^{\circ} 26^{\prime} \mathrm{N}, 1^{\circ} 4^{\circ} 07^{\prime} \mathrm{W}, 6$ ago. 1981, González y Acevedo 1825 (CHAPA, CIIDIR, IEB, MEXU, TEX); entrada al rancho El Cordón Santo, cerca del Cerro La Zorra, González 3762 (CIIDIR, MEXU); Magueycitos, $20 \mathrm{~km}$ al SW de Rancho de Peña, Martínez 597 (MEXU); Magueycitos, 2 jun. 1975, Maury et al. 31 (CIIDIR, IEB, MEXU); Mpio. Tepehuanes, camino Tepehuanes-El Tarahumara, Benitez 937 (CIIDIR, MEXU); Sierra Madre Occidental, $19 \mathrm{mi} \mathrm{W}$ of Ojito, Reveal \& Hess 3075 (MEXU). GUANAJUATO: Mpio. Dolores Hidalgo, carretera Dolores HidalgoGuanajuato, km 83, Carranza 4057 (IEB, MEXU, XAL); carretera Guanajuato-Dolores Hidalgo, km 85, Martínez 1011 (MEXU); Mpio. Guanajuato, Cañada La Virgen, Cano 02 (CIIDIR, IEB, MEXU, XAL), 09 (IEB, MEXU); carretera Dolores Hidalgo-Guanajuato, km 38, Kishler 760 (MEXU); camino a Agua Sabrosa-Viborillas, Martínez 426 (MEXU); La Mesa de Hernández, Cardo Santo, Martínez 648 (MEXU), Martinez 820 (MEXU); El Ciervo, Martínez 959 (MEXU); Presa Peralvi1lo, Zamudio \& Murillo 6387 (CIIDIR, IEB, MEXU, XAL); Santa Rosa de Lima, 5 sept. 1998, Zamudio \& Murillo 10820 (CIIDIR, IEB, MEXU, XAL); HIDALGO: Mpio. Epazoyucan, $1 \mathrm{~km}$ al S de Peñas Largas, 1 sept. 1963, Rzedowski 30530 (ENCB, MEXU); El Guajolote, 2006' N, 98³6' W, 17 jun. 1984, Medina \& Barrios 2576 (ENCB, MEXU, TEX); Mpio. Zimapán, $38 \mathrm{~km}$ al NE, Hernández 3884 (MEXU); NUEVO LEÓN: Mpio. Aramberri, Cañón Los Capulines, San José de Raices, 6 ago. 1935, Muller 736 (MEXU, TEX); Mpio. Doctor Arroyo, $46 \mathrm{~km}$ al N de Doctor Arroyo, 22 jun. 1970, Flyr 1507 (TEX); San Antonio Peña Nevada, 24 may. 1992, Hernández 2735 (TEX); San Antonio Peña Nevada, 2348' N, 9953' W, jul. 1977, Wells \& Nesom 368 (TEX); Mpio. Galeana, Cerro El Potosí, 20 jun. 1972, 
Chiang et al. 8062 (MEXU, TEX); 2 miles S of Pablillo, 20 jul. 1958, Correll \& Johnston 19890 (ASU, MEXU, TEX); cima Cerro Potosí, 2453' N, 100²' W, 12 may. 1982, Dorr \& Atkins 2299 (MEXU, TEX, XAL); San Pedro Sotolar, pine oak wood, 31 may. 1983, Dorr et al. 2646 (MEXU, TEX, XAL); camino Los Mimbres a San Rafael, 1950 m, matorral, 30 jun. 1991, Estrada et al. 2045 (ANSM); 4 km al N del ejido Cieneguillas, bosque de Pinus cembroides, 12 jul. 1979, Flores s.n. (ANSM); Cerro El Potosí, clearing in mixed forest, 3190 m, 22 jun. 1969, Hinton 17127 (TEX); Cerro El Voladero, 3 jul. 1984, Hinton 17249 (TEX); Pablillo, 5 may. 1983, Hinton 17370 (CHAPA, IEB, MEXU); El Potosí, 22 jun. 1969, Hinton 18724 (TEX); Cerro El Potosí, open forest, 8 jul. 1963, McGregor 341 (TEX); 15 mi SW of Galeana, 15 jun. 1934, Muller 800 (MEXU, TEX), Muller 2398 (MEXU, TEX); camino del ejido 18 de Marzo al Cerro del Potosí, Yanéz \& McDonald 224 (MEXU); Pablillo, 28 may. 1969, Seigler 1364 (TEX); áreas cercanas a Pablillo, 2430' N, $100^{\circ} 05^{\prime} \mathrm{W}, 2100 \mathrm{~m}$, bosque de pino piñonero, 12 jun. 1985, Villarreal et al. 3034 (ANSM, CIIDIR, MEXU, TEX); San José de la Marta, 2510' N, 100¹5' W, 1900 m, bosque de pino piñonero, 7 junio 1988, Villarreal 4328 (ANSM, TEX); Mpio. Montemorelos, El Butano, 2000 m, bosque de coníferas, 3 jun. 2004, Estrada et al. 16344 (ANSM); Mpio. Rayones, cima de la Sierra de la Marta, 2509' N, 100 $23^{\prime}$ W, 3400 m, bosque de Pinus hartwegii, 19 may. 1995, Villarreal et al. 8132 (ANSM); Mpio. Zaragoza, Cerro El Viejo, oak and pine wood, 2380 m, 29 jul. 1993, Hinton 23262 (IEB, TEX); Cerro el Viejo, 2400 m, Cedar forest, 4 ago. 1993, Hinton 23320 (ANSM, IEB, TEX); Cañada La Tinaja, 2356' N, 9949' W, 3 july 1988, Patterson 5776 (TEX). OAXACA: Mpio. San Miguel del Puerto, Llano del Horno, López 141 (MEXU). PUEBLA: Plantas del parque estatal General Lázaro Cárdenas, Martínez 365 (MEXU), 718 (MEXU). QUERÉTARO: Mpio. Cadereyta, alrededores de El Doctor, Hernández et al. 11019 (MEXU); alrededores de Chavarría, Huerta 1505 (MEXU, XAL); $3 \mathrm{~km}$ al S de Chavarrias, $2800 \mathrm{~m}$, bosque de pino piñonero, 19 jul. 1990, Zamudio 7929 (ANSM, IEB, MEXU); Chavarría, Sierra El Doctor, Zamudio \& Carranza 6433 (IEB); Mpio. Jalpan, 4-5 km al poniente de La Parada, Servín 325 (IEB, MEXU); Mpio. Landa, $1 \mathrm{~km}$ al SW de El Sabinito, Rubio 1158 (CIIDIR, IEB, XAL); $1.5 \mathrm{~km}$ al NE de El Sabinito, Rubio 1243 (IEB); $1.5 \mathrm{~km}$ al SE de La Yesca, Rubio 2418 (IEB); 6-7 km al NE de Lagunita de San Diego, Rubio 2577 (IEB, MEXU); Mpio. Pinal de Amoles, al SW de El Madroño, Carranza 2694 (IEB); Mpio. San Joaquín, Campo Alegre, Fernández 3296 (IEB, TEX). SAN LUIS POTOSÍ: Mpio. Charcas, Sierra de Monte Grande, Reyes 823 (CHAPA); Sierra de Monte Grande, ladera Norte, Reyes 917 (CHAPA); Ex convalli San Luis Potosí, Schaffner 733 (MEXU); TAMAULIPAS: Mpio. Gómez Farías, El Paraiso, Rancho El Cielo, 
González et al. 7502 (ANSM, MEXU); rancho El Julilo, $1800 \mathrm{~m}$, bosque caducifolio, 25 sept. 1974, González et al. 7503 (ASU, MEXU); El Paraiso, 7 km al W del Rancho El Cielo, González et al. 7504 (ANSM, MEXU); Mpio. Jaumave, $15 \mathrm{~km}$ al N de Julilo, González \& Martínez 3661 (MEXU); Mpio. Miquihuana, $17 \mathrm{~km}$ al NE de La Peña, González et al. 7051 (MEXU). ZACATECAS: Mpio. Genaro Codina, carr. Guadalupe-Pabellón, km 80, Balleza 16802 (MEXU); Sierra del Astillero, Tanque El Alto, 2434' N, 10104' W, 2 jul. 1973, Johnston et al. 11553 (MEXU, TEX).

Lonicera pilosa (Kunth) Spreng var. glabra Villarreal, A. E. Estrada \& A. D. Ruíz, var. nov. Tipo: MÉXICO. Durango, municipio de Mezquital, Ojo de Agua, $20 \mathrm{~km}$ de La Escondida por el camino a Los Charcos. 18.06.85. S. González et al. 1755 (Holotipo: MEXU; isotipos: ANSM, CIIDIR, CHAPA).

Similar to Lonicera pilosa var. pilosa but flowers with peduncles and calyces glabrous; calyx neck 1.5-2.0 mm long; corolla externally glabrous, internally pilose.

Flores con pedúnculo y cáliz glabros; cáliz con cuello 1.5 a $2.0 \mathrm{~mm}$ largo; corola glabra externamente y pilosa internamente (Fig. 3B).

Distribución: Sierra Madre Occidental y Faja Volcánica Transmexicana (Fig. 2).

Ejemplares examinados: MÉXICO. CHIHUAHUA: Mpio. Guachochi, Norogachi, 15 jul. 1955, Pennington 195 (TEX); Llano Grande, 23 may 1960, Pennington 586 (TEX); Mpio. Guazapanes, on slope E and above Las Lagunitas, barranca de Chinipas, Bye 6581 (MEXU); La Ciénega, 19 jul. 1965, Pennington 64 (TEX); Mpio. Madera, arroyo La Quinta, ejido El Largo, 2100 m, bosque de pino-encino, 13 oct. 1990, Benitez 2851 (ANSM, CIIDIR, IEB, MEXU); arroyo Las Garrochas, Ejido El Largo, 2600 m, 2 jul. 1990, Bravo 921 (ANSM, CIIDIR, IEB, MEXU); $29 \mathrm{~km}$ E of Yepachic along road to Chihuahua, Breedlove 61212 (MEXU); Cerro Mohinora, 10 miles S of Guadalupe y Calvo, Straw \& Forman 2038 (MEXU); Mpio. Ocampo, Basaseachi, 26 sept. 1986, Torres \& Tenorio 3810 (MEXU, TEX). DISTRITO FEDERAL: Del. Álvaro Obregón, Sector Limbo, Ventura 2666 (MEXU); San Bartolo, 2700 m, matorral, 25 abril 1977, Ventura 2698 (ASU, ENCB, MEXU); Santa Rosa, Ventura 2817 (IEB, MEXU), 2718 (ASU, CHAPA, MEXU); Del. Cuajimalpa, Los Gavilanes, Cesar \& Rivera 298 (MEXU); Desierto de Los Leones, 19¹9' N, 99¹8' W, 23 mar. 1991, Chávez s.n. (ANSM); Desierto de Los Leones, Lyonnet 1125 (ENCB, MEXU), 2135 (CHAPA, MEXU), 3077 (IEB, MEXU); Desierto de Los Leones, Paray 491 (ENCB, 
MEXU); Thickets near Eslava, Pringle 9380 (MEXU), 13009 (MEXU); Desierto de Los Leones, Sabandijas, Rivera 4122 (MEXU); W of la Marquesa in Desierto de Los Leones, Wonderly 203 (MEXU); Del. Magdalena Contreras, Cañada de Contreras, Rivera et al. 3931 (MEXU); Del. Tlalpan, Pedregal de San Ángel, cerca de San Ángel, matorral, 27 de oct. 1966, Diego 1200 (ENCB, XAL); Panteón Jardín, Ulloa s. $n$. (MEXU). DURANGO: paraje Arroyo Boscoso, $1 \mathrm{~km}$ antes de El Tarahumara, Benitez 470 (CIIDIR, MEXU); Mpio. Canelas, Cuevecillas, 26 sept. 1990, Bravo 30 (CIIDIR, IEB, MEXU); Route 40, ca $9 \mathrm{~km}$ E of El Salto, Cruden 1304 (MEXU); Mpio. Mezquital, $3 \mathrm{~km}$ al W de Santa María Ocotlán, bosque de pino, 15 ago. 1984, González 1409 (ANSM, CIIDIR, MEXU); Ojo de Agua, $20 \mathrm{~km}$ de La Escondida, $2719 \mathrm{~m}$, bosque de pino-encino, 18 jun. 1985, González et al. 1755 (ANSM); $8 \mathrm{~km}$ de La Guajolota, bosque de encino, 29 jul. 1985, Solís 41 (CHAPA, IEB), Solís 57 (ANSM, CIIDIR); La Guajolota, 2257' N, 104³7’ W, 29 jul. 1985, Solís 916 (CHAPA, CIIDIR, IEB); Mpio. Nuevo Ideal, Sierra de Coneto, $24^{\circ} 55^{\prime}$ N, 104 $46^{\prime}$ W, 2280 m, bosque de Pinus-Quercus, 25 may. 2002, López et al. 121 (ANSM, CIIDIR, MEXU); Hacienda Coyotes, 63 road miles W-SW of Durango, Maysilles 7485 (MEXU); Mendía 14 (CIIDIR); Mpio. Pueblo Nuevo, Corralitos, 2325' N, 105²2' W, 26 sept. 1982, Fernández 1224 (CIIDIR, IEB); alrededores del Mil diez, 2 km al NW de El Salto, Hernández \& Tenorio 7505 (MEXU); 46 km de la desviación a San Miguel de Cruces, Hernández et al. 7745 (MEXU); Mpio. Santiago Papasquiaro, Garame, 26 km al W de Santiago Papasquiaro, 1800 m, bosque de pino piñonero, 15 jul. 1982, Hernández et al. 7910 (ANSM, MEXU), 7923 (MEXU, XAL). JALISCO: Mpio. Ayutla, 19 km WSW de La Ferrería, 1957' N, 104²2' W, 1800 m, 12 ago. 2012, Carrillo \& Monroy 6739 (ANSM); Mpio. Tecatitlán, La Resbalosa, Pichardo 122 (CHAPA); $7 \mathrm{mi}$ W of Tecatitlán, Rodman \& Schnell 10 (MEXU); Mpio. Tolimán, El Timbre, cerca de Picachos, Cerro Grande, Sánchez \& Cruz s.n. (MEXU); Camino del Tuito a Tlapa, después de la mina, Téllez 6814 bis (MEXU, XAL). MÉXICO: Mpio. Huixquilucan, ladera húmeda en bosque abierto, Matuda 21570 (MEXU); Mpio. Jilotepec, encinar cercano a Coscomate, Villaseñor 06 (MEXU); Mpio. Jilotzingo, Cerro Naadó, al N de San Luis Ayucan, Rzedowski 33988 (ENCB, MEXU); Cerro Venacho, al E de Amecameca, Rzedowski 33972 (MEXU); San Francisco Acuautla, Urbina s.n. (MEXU); Mpio. Nicolás Romero, Colonia Libertad, 2300 m, matorral, 21 oct. 1978, Ventura 3363 (ASU, ENCB, MEXU, XAL); Mpio. San Felipe del Progreso, orilla de arroyo, Matuda 19742 (MEXU); San Luis Ayucan, Matuda et al. 28289 (MEXU); Barranca Papaloaque, bajando a San Rafael, Miranda 261 (MEXU); Mpio. Temascaltepec, Cajones, 22 may. 1933, Hinton 3867 (ASU, DES); La Cumbre, Gavia Chica, 12 abr. 1936, Hinton et al. 9010 (TEX); Mpio. Texcoco, 9 km al SE de Tequexquinahuac, bre- 
cha a la cima del Cerro Tlaloc, Charles \& Perino 3433 (CHAPA); Sierra Tlaloc, García 1795 (CIIDIR, MEXU, TEX); San Pablo Ixyoc, Hahn 552 (MEXU); Cerro Tlaloc, Koch \& Koch 9512 (CHAPA); Texcoco, 9 km al S de Tequexquinahuac, Cerro Tlaloc, Perino 3433 (CHAPA, ENCB); Santa Catarina del Monte, 8 jun. 1985, Ventura 4413 (ENCB, IEB, MEXU, TEX); Mpio. Valle de México, Cerro de León, Matuda 19120 (MEXU). MICHOACÁN: Mpio. Nahuatzen, Cerro Pelón, 6 dic. 1985, Martínez 410 (IEB, MEXU); salida poniente de Sicuicho, Motte 240 (MEXU); MORELOS: Parque Nacional Lagunas de Zempoala, Smith 51 (MEXU ); Lagunas de Zempoala, Vázquez 3217 (MEXU). NAYARIT: Mpio. Acaponeta, La Cienega, $10 \mathrm{mi}$. NW of Mesa del Nayar, Norris \& Taranto 14608 (MEXU). SINALOA: Mpio. Concordia, $50 \mathrm{~km}$ al NE de Concordia, carr. Mazatlán-Durango, Ortíz et al. 270 (MEXU); El Palmito, Vega 1426 (CHAPA, MEXU). SONORA: Mesa El Campanero, Arroyo Largo, Van Devender et al. 410 (MEXU); Mpio. Yécora, Mesa El Campanero, barranca El Salto, 14 jul. 1997, Van Devender 97-701 (TEX). ZACATECAS: Mpio. Teul de González

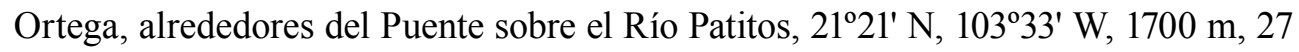
may. 2015, Carrillo \& Ramírez 7818 (ANSM).
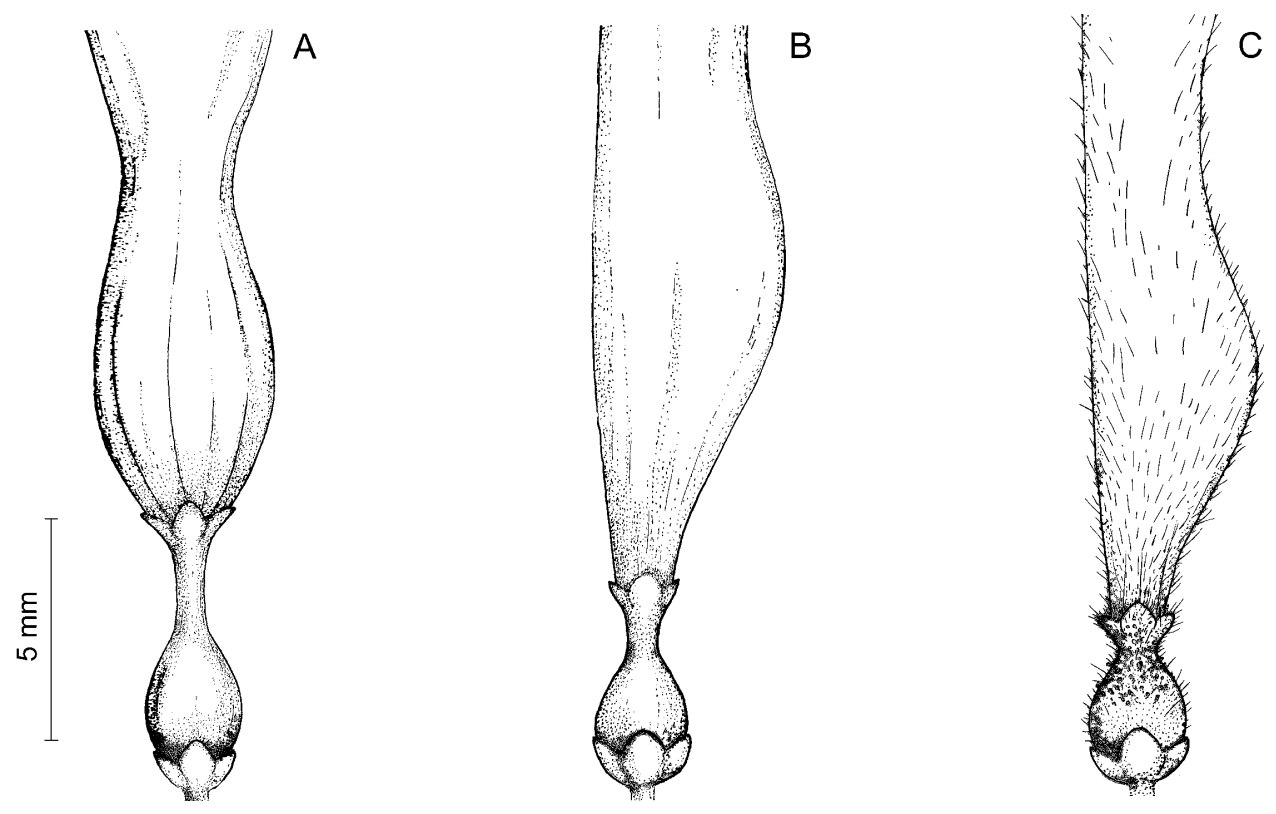

Fig. 3. Detalle de las flores en A. Lonicera cerviculata, B. L. pilosa var. glabra, C. L. pilosa var. pilosa. Ilustrado por Gabriel Gloria. 


\section{AGRADECIMIENTOS}

A los curadores de los herbarios citados y a los revisores anónimos. Al M.C. Félix Sánchez Pérez por su ayuda con el análisis estadístico. Por el apoyo financiero otorgado por el CONACYT, a través de la beca 545819 a la estudiante Alma D. Ruíz A. A la Universidad Autónoma Agraria Antonio Narro, que patrocinó mediante el proyecto 3614-2108, titulado Plantas endémicas y raras de Coahuila.

\section{LITERATURA CITADA}

Chauza-López, L. J. y E. A. Villa-Villada. 2011. Análisis de conglomerados comparando el coeficiente de similaridad de Gower y el método análisis factorial múltiple para el tratamiento de tablas mixtas. Escuela de Ingeniería Industrial y Estadística. Facultad de Ingeniería. Universidad del Valle. Santiago de Cali, Colombia. 72 pp.

De la Fuente-Fernández, S. 2011. Tablas de contingencia, análisis de variables categóricas. Facultad de Ciencias Económicas y Empresariales. Universidad Autónoma de Madrid. Madrid, España. 52 pp.

Gower, J. 1967. A comparison of some methods of Cluster Analysis. Biom. J. 23: 623-637.

Greenacre, M. 2007. Correspondence analysis in practice. Second edition. Chapman \& Hall. London, U.K. 311 pp.

Johnson, R. A. y D. W. Wichern. 2007. Applied multivariate statistical analysis. Sixth edition. Prentice-Hall. New Jersey, USA. 767 pp.

Linares, G. 2001. Escalonamiento multidimensional: conceptos y enfoques. Rev. Inv. Oper. 22(2): 173-183.

Meulman, J. J. y W. J. Heiser. 2010. IBM SPSS Categories 19. SPSS Inc. Chicago, USA. 318 pp.

Rehder, A. 1903. Synopsis of the genus Lonicera. Ann. Rep. Mo. Bot. Gard. 14: 27-232.

Theis, N., M. J. Donoghue y J. Li. 2008. Phylogenetics of Caprifolieae and Lonicera (Dipsacales) based on nuclear and chloroplast DNA sequences. Syst. Bot. 33(4): 776783.

Thiers, B. Continuously updated. Index Herbariorum: A global directory of public herbaria and associated staff. New York Botanical Garden's Virtual Herbarium. http:// sweetgum.nybg.org/science/ih/.

Villarreal-Quintanilla, J. Á. 2000. Caprifoliaceae. Flora del Bajío y de Regiones Adyacentes 88: $1-16$.

White, S. S. 1947. New plants from northeastern Sonora, Mexico and notes on extensions of range. J. Arnold Arb. 28: 439-441. 